\title{
Altered Chondrocyte Apoptosis Status in Developmental Hip Dysplasia in Rabbits
}

\author{
Yi-Shan Wei ${ }^{1,2}$, Dai-He Li ${ }^{2}$, Wan-Lin Liu ${ }^{2}$, Dian-Ming Jiang ${ }^{1}$ \\ ${ }^{1}$ Department of Orthopedics, First Affiliated Hospital, Chongqing Medical University Chongqing, China \\ ${ }^{2}$ Department of Orthopedics, The Second Affiliated Hospital of Inner Mongolia Medical University, Hohhot, China
}

Background: Developmental dysplasia of the hip $(\mathrm{DDH})$ is an important factor leading to early adult osteoarthritis. Chondrocyte apoptosis has been proven to be an important factor causing osteoarthritis.

Aims: The current study aims to explore whether a rabbit model of developmental dysplasia of the hip through cast immobilization in the legs results in chondrocyte apoptosis.

Study Design: Animal experimentation.

Methods: Thirty-two New Zealand white rabbits were divided in three groups with cast plaster-induced dislocation at 2, 4 and 6 weeks. The contralateral hip joint was utilized as a control group. Ten rabbits in each group were sacrificed, and hip specimens were obtained. Bcl-2/Bax, cleaved caspase- 3 and cleaved caspase- 8 expression were examined by western blot analysis. Chondrocyte apoptosis was analyzed through transmission electron microscopy (TEM) and
TUNEL analysis. All experiments were repeated at least three times.

Results: In the experimental group, Bcl-2/Bax, cleaved caspase- 3 and cleaved caspase- 8 expression were significantly altered. The $\mathrm{Bcl}-2 / \mathrm{Bax}$ ratio decreased with time (all $\mathrm{p}<0.01$ ), whereas levels of cleaved caspase- $3(\mathrm{p}<0.01$ and $\mathrm{p}<0.05)$ and cleaved caspase-8 (all $\mathrm{p}<0.05)$ gradually increased. Chondrocyte apoptosis was observed through transmission electron microscopy (TEM) and TUNEL analysis $(\mathrm{p}<0.05$ at 4 weeks and $\mathrm{p}<0.01$ at 6 weeks $)$.

Conclusion: Prolonged immobilization of rabbit hip caused chondrocyte apoptosis. Reduction of the hip joint may protect chondrocytes from apoptosis, thus preventing secondary osteoarthritis.

Keywords: Developmental dysplasia of the hip, chondrocyte apoptosis, immoblilization
Developmental dysplasia of the hip (DDH) is a spectrum of hip joint abnormalities ranging from mild temporary instability to frank dislocation (1). The overall DDH risk is about 1 per 1000 according to the statistics (2) Mild cases of DDH usually resolve spontaneously. However, late diagnosis of DDH probably result in early development of degenerative osteoarthritis (OA), which may cause great burdens to the patients in the future (3). A registry based study demonstrated that $29 \%$ of primary hip replacements in people under 60 years was attributed to DDH (4). Although the detailed etiology of DDH remains unknown, genetic and environmental factors may serve as internal or external components respec- tively (5-6). It is believed recently that rather than congenital factors, acquired influences may play more important roles in the progression of DDH (7), implicating that most cases of DDH appear gradually with growth and could be prevented.

Increasing amounts of evidence has shown that chondrocyte apoptosis play a great role in cartilage development, aging and disease (8-9). Chondrocyte apoptosis is of great clinical significance as it often occurs in secondary osteoarthritis (10-11). It has been proven that chondrocyte death may accelerate the progression of osteoarthritis (12). In addition, the localization of chondrocyte apoptosis near the lesion area of osteoarthritic cartilage further emphasizes the importance of chondrocyte 
death in the progression of OA (13-14). To prevent chondrocyte apoptosis may benefit cartilage metabolism, thus obtaining favorable prognosis of DDH.

To our knowledge, the fate of articular cartilage following chondrocytes following DDH model, has not been documented. Therefore, the aim of the current study is to investigate the changes of chondrocyte apoptosis in hip cartilage during the cast plaster-induced joint dislocation in a rabbit DDH model.

\section{MATERIALS AND METHODS}

\section{Materials and modeling}

The study was performed in conformity with the ethical guidelines of the Declaration of Helsinki. This program was approved by School of Medicine Animal Care and Investigational Committee in our institution. Three groups of male, 4-week old New Zealand rabbits weighing from 450 to 550 $\mathrm{g}$ were used. There were 12 rabbits with hips dislocated for 2 weeks and 10 rabbits each in two groups with hips dislocated using cast plaster for 4 weeks and 6 weeks, respectively. In brief, the lower body, hips and the limbs were immobilized with the hip flexion of $120^{\circ}$, abduction of $60^{\circ}$ and knee joint extension. The contralateral hip joint was utilized as control group (dislocation followed by immediate reduction). Animals were anaesthetized using 5\% intramuscular hydrochloride ketamine. The initial dose given was $1 \mathrm{mg} / \mathrm{mL}$ bodyweight. The rabbits' respiratory rate and reaction were monitored simultaneously in the process of modeling. Success of DDH modeling was identified through the presence of increased acetabular index, discontinued Shenton line, and dislocation of the femoral head according to the X-ray plain.

\section{Tissue pretreatment}

Acetabular cartilage tissues were obtained in each group at 2, 4, 6 weeks, respectively. Cartilage tissues for western blot were directly stored in $-80^{\circ} \mathrm{C}$ until lysis. The remaining cartilage tissues were fixed in $10 \%$ formalin and stored at $4^{\circ} \mathrm{C}$ for 10 days. Specimens were then decalcified in a routine prices for 3-4 weeks at $4^{\circ} \mathrm{C}$. The solution was changed twice per week. Specimens were then cut and embedded in paraffin. Embedded specimens were sliced into 5-lm-thick slices in the coronal plane and stained with hematoxylin and eosin.

\section{Morphological changes observation by transmission electron microscopy}

Apoptotic morphology of chondrocytes can be observed directly using transmission electron microscopy (TEM). Briefly, the acetabular cartilage tissues were fixed and then decalcified as described previously (15). Then they were embedded and cut into sections stained with uranyl acetate and lead citrate. The sections were viewed in a transmission electron microscope at $80 \mathrm{kV}$ (Philips; Amsterdam, Holland).

\section{TUNEL assay}

After the pretreatment process, the slides were stained utilizing in situ cell death detection POD kit (Roche; Penzberg, Germany), based on the manufacturer's procedure. Prior to diaminobenzidine (DAB) coupling. Each slide was examined in five fields at $\mathrm{x} 400$ magnification and read three times by two pathologists. Then, the mean number of positive chondrocytes was divided by the total number of chondrocytes to calculate the TUNEL-positive chondrocyte ratio.

\section{Western blot analysis}

Western blot was performed by the chemiluminescence method. Protein concentration was determined by the bicinchoninic acid assay (BCA) method. The membranes were first blocked with $5 \%$ milk and then incubated respectively with mouse anti-actin (Chemicon; Billerica, MA, United States); rabbit anti-cleaved-caspase-3 (Chemicon), cleaved caspase-8 (Chemicon), rabbit anti- Bcl-2 (Santa Cruz Biotechnology; Dallas, TX, United States) and rabbit anti-Bax (Santa Cruz). Immunoreactive reactions were assessed using ECL system and quantified by designated software.

\section{Statistics}

All data were demonstrated as the mean \pm SD. Statistical Package for the Social Sciences version 16.0 for Windows (SPSS Inc.; Chicago, IL, USA) was utilized for statistical analysis. Kolmogorov-Smirnov test was used test normality distribution of all expression values of apoptosis rate and tested apoptosis related proteins. One-way analysis of variance (ANOVA) were applied to compare the differences between groups. Bartlett's test was used to test the homogeneity of group variances, followed with Tukey or Tamhane post-hoc tests, where appropriate. The statistical power was also calculated. A $p$ value of $<0.05$ was regarded statistically significant.

\section{RESULTS}

\section{Radiographic changes}

To monitor the whole process of modeling, we accordingly performed X-ray plain to observe the dislocation of hip. The Xray before modeling was regarded as control group (Figure 1a). 


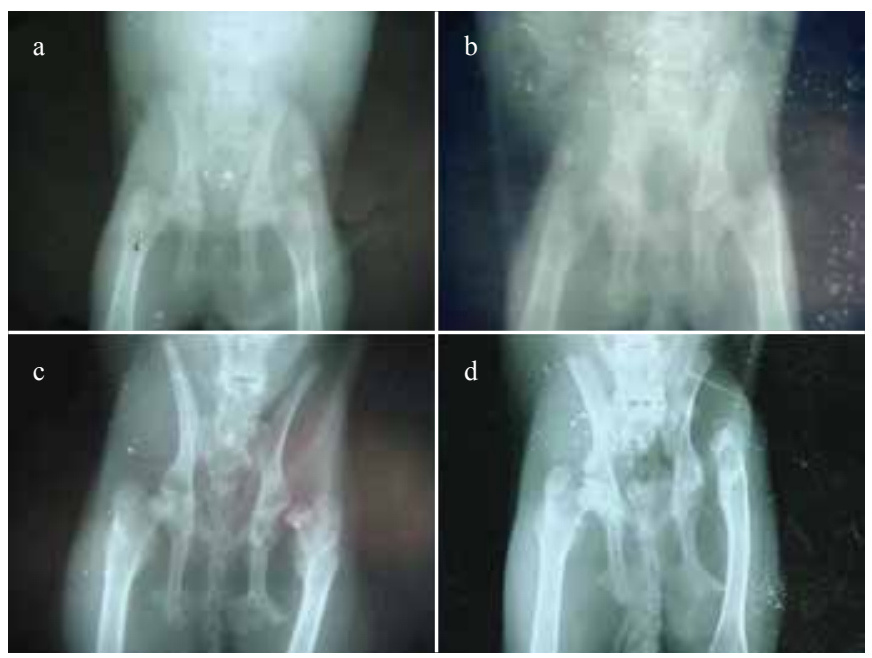

FIG. 1. a-d. Control Group (a), 2-week Group (b), 4-week Group (c), 6-week Group (d). Gradual dislocation with blunt and straight superior border of acetabulum and disappearing Shenton line in the experimental group following immobilization at 2, 4, 6 weeks compared with control group.

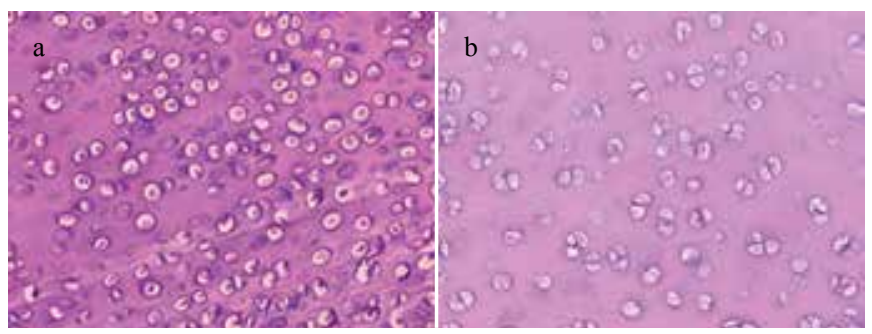

FIG. 2. a-b. Control Group (a), 6-week Group (b). Hematoxylin and eosin stain of acetabular cartilage $(\times 100$ magnification). Comparison between the control (a) and 6-week groups (b) showing significant difference in histological appearance of the articular cartilage.

In the first 2 weeks, dysplasia without subluxation exists could be observed. The head is insufficiently covered by the acetabulum, without proximal displacement of the proximal femur (Figure 1b). In the 4-week group, the head and acetabulum began to display partial contact (subluxation) (Figure 1c). In the 6-week group, there was no contact existing between the femoral head and acetabulum with the broken Shenton's line (dislocation) (Figure 1d).

\section{HE staining and TUNEL analysis}

Hematoxylin and eosin staining demonstrated that there was significant difference in appearance of chondrocyte and cartilage thickness between the 6-week group and control group (Figure 2a, b). For TUNEL analysis, there is an increased number of apoptotic nuclei in the 4-week group and 6-week group compared with the control group respectively $\left({ }^{*} \mathrm{p}<0.05\right.$ and $\left.* * \mathrm{p}<0.01\right)$. Although the number of apoptotic nuclei was increased in the 2-week group as compared with control, statistics did not reach significant difference $(\mathrm{p}>0.05)$ (Figure 3a-e).

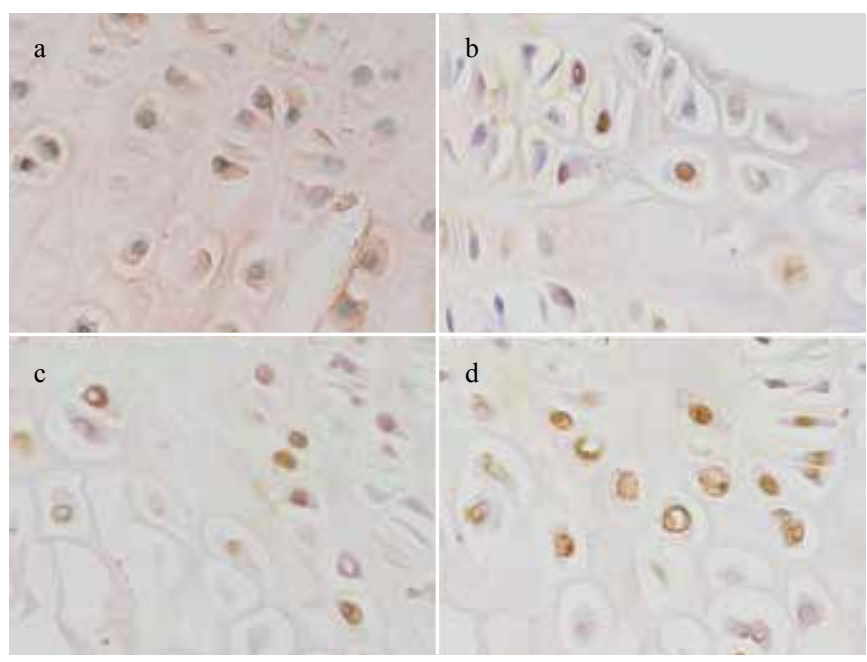

e

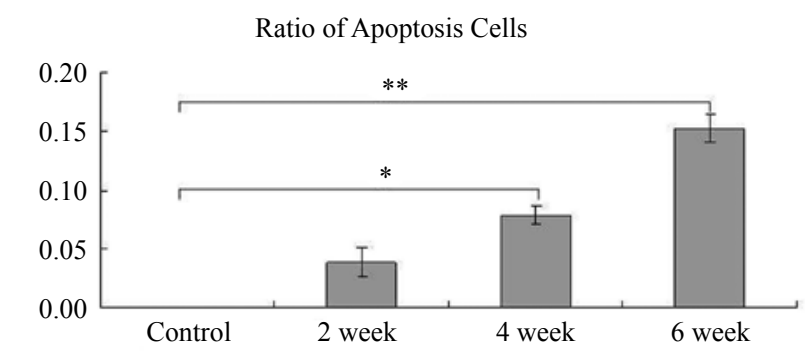

FIG. 3. a-e. Control Group (a), 2-week Group (b), 4-week Group (c), 6-week Group (d), Quantified analysis of acetabular cartilage apoptosis ratio TUNEL stain of acetabular cartilage Comparison among the control, 2-week immobilization group, 4-week immobilization group and 6-week immobilization group (400 magnification) (e). TUNEL, terminal deoxynucleotidyl transferase-mediated dUTP nick-end labelling. Values are shown as mean \pm standard deviation. The error bars represent the standard deviation of the mean. ${ }^{*} \mathrm{p}<0.05,{ }^{*} \mathrm{p}<0.01$ versus control group.

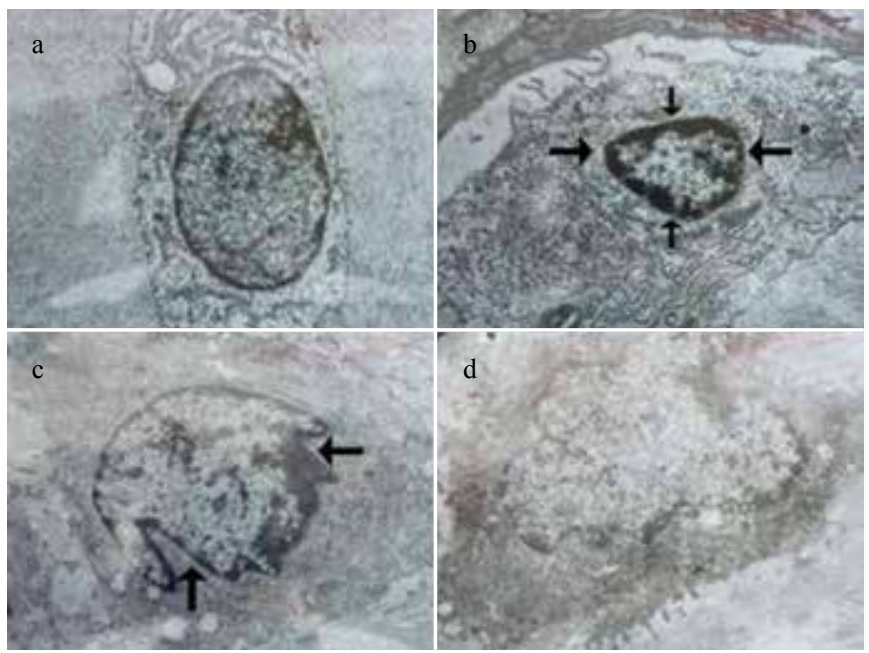

FIG. 4. a-d. Control Group (a), 2-week Group (The arrows indicate cellular shrinkage) (b), 4-week Group (The arrows indicate nuclear fragmentation, condensed chromatin and reduced organelles) (c), 6-week Group (The figure indicates the apoptosis) (d). 


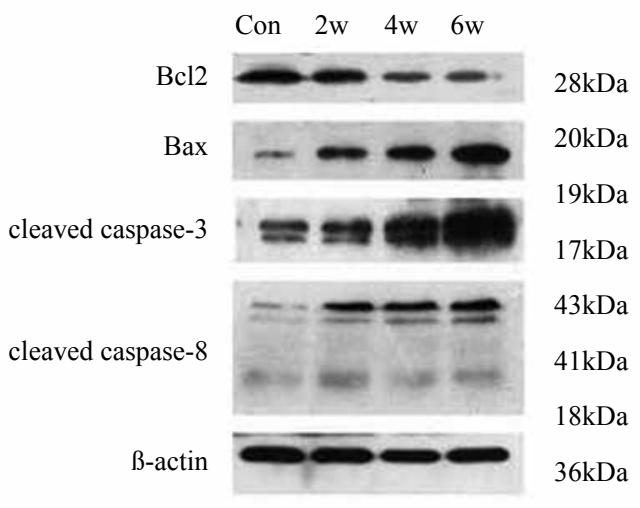

b
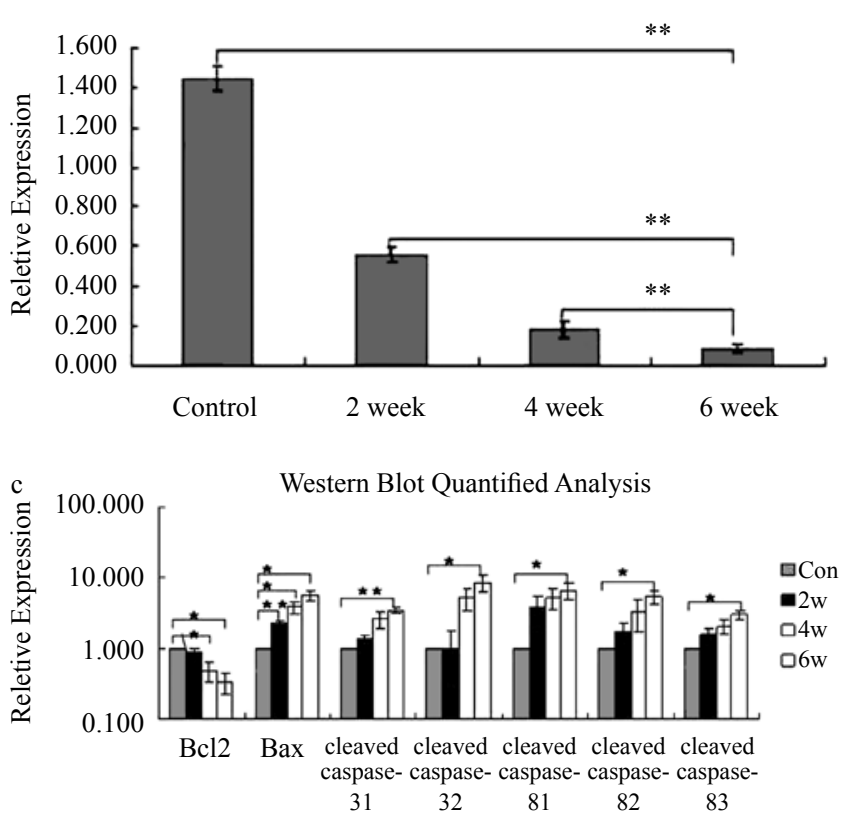

FIG. 5. a-c. Expression of apoptosis related proteins by western blot analysis (a), Quantified analysis of Bcl-2/Bax Ratio. Expression of Bcl-2 plays an apoptosis-inhibiting role, whereas the expression of Bax plays a contrary role (b), quantified analysis of relative expression (c). Values are shown as mean \pm standard deviation. The error bars represent the standard deviation of the mean. ${ }^{*} \mathrm{p}<0.05, * * \mathrm{p}<0.01$ versus control group.

\section{TUNEL analysis}

For TUNEL analysis, there is an increased number of apoptotic nuclei in the 4-week group and 6-week group as compared with the control group respectively $\left({ }^{*} p<0.05\right.$ and $* * p<0.01)$. Although the number of apoptotic nuclei was increased in 2-week group compared with control, statistics did not reach significant difference $(\mathrm{p}>0.05$ ) (Figure 3a-e).

\section{Transmission electron microscopy}

In the control group, the normal acetabular chondrocytes were round and normal, with an entire membrane as well as abundant figure and evenly distributed chromatin (Figure 4a).
However, in the experimental side, acetabular chondrocytes were shown at 2, 4 and 6 weeks with manifestations of apoptosis (Figure 4b-d).

\section{Apoptosis related protein assessment}

Western blot analysis was used to assess the expression levels of several apoptosis related proteins to determine the chondrocytes apoptosis rate. Following 2-, 4- week and 6-week immobilization, protein levels of Bcl-2, Bax, Caspase 3 and Caspase 8 were detected. The results revealed significantly reduced expression $\mathrm{Bcl}-2 / \mathrm{Bax}$ ratio $(* * \mathrm{p}<0.01)$, whereas Caspase3, and Caspase8 were gradually increased in the experiment groups $\left({ }^{*} \mathrm{p}<0.05,{ }^{* *} \mathrm{p}<0.01\right)$ (Figure $\left.5 \mathrm{a}-\mathrm{c}\right)$.

\section{DISCUSSION}

The current study focused on acetabular chondrocyte apoptosis in a rabbit model of cast plaster immobilization. We demonstrate from different aspects that prolonged immobilization of rabbit hip caused chondrocyte apoptosis. The apoptotic degree after joint immobilization is in a time-dependent manner. The joints that remained dislocated at 6 weeks exhibited the highest apoptotic index. These findings made clinical significance as they implicated that chondrocytes apoptosis is closely related to continuous joint dislocation.

The most widely used term of DDH in the past was the acronym: congenital dislocation of the hip $(\mathrm{CDH})(16)$. It has gradually been recognized that this disorder is not always congenital (17). The change of naming and definition of this disorder indicates that acquired influences may play pivotal parts in the disease progression.

Chondrocyte apoptosis has been demonstrated to be engaged in the pathogenesis of OA. Human and animal studies of OA have shown that chondrocyte death by apoptosis is at least partly responsible for reduced cellularity, cartilage breakdown and matrix turnover abnormalities (18). Therefore, chondrocyte apoptosis may be not only the result of disorder, but also a factor causing vicious cycle in disease progression.

In the present study, we utilized cast immobilization model with hip flexion and knee joint extension as DDH modeling, which renders the hip joint dislocated. We first used transmission electron microscopy and the TUNEL assay to examine apoptosis and found morphological changes with signs of apoptosis and increased chondrocyte death. Next, we performed western blot analysis of several apoptosis related proteins. Previous studies have demonstrated that the expression of Bcl-2 plays an apoptosis-inhibiting role, whereas the expression of Bax plays a contrary role $(19,20)$. The expression balance of Bcl-2/Bax determines death or 
survival of the cells. In this study, the rate of apoptotic chondrocytes was in consistent with the decreased ratio of Bcl-2/ Bax expression. The caspase family of enzymes also plays a pivotal role in the process of cell apoptosis (21). Caspase-8 is an initiator caspase to cleave inactive pro-forms of effector caspases, thereby activating them to trigger apoptosis (22). It is activated in response to agents or insults inducing the release of cytochrome from the inner mitochondrial membrane (23), playing a crucial role in generating cell apoptosis downstream pathway (24). Caspase-3 is required for cell deaths and distinct apoptotic processes (25). Generally, caspase- 3 is activated by initiator caspases such as caspase- 8 and cleaves important vital intracellular proteins (25). Here, we also found caspase- 3 and caspase- 8 expression elevated as immobilization prolonged.

One limitation should be pointed out in our study. After calculation, the statistical power is $0.73<0.80$. Perhaps this is attributed to the small cases of animals we utilized. Therefore, further larger size of samples should be used to enhance the statistical power.

Collectively, we observed excessive chondrocyte apoptosis in the process of cast immobilization in early and middle phase, which emerges as an important factor leading to the degeneration of the acetabular cartilage and accelerate the development of disease. To prevent the chondrocyte apoptosis or to remove factors (like subluxation or dislocation) resulting in chondrocyte apoptosis in the early phase may serve as a potential way to promote the acetabular dysplasia.

Ethics Committee Approval: Ethics committee approval was received for this study from the ethics committee of First Affiliated Hospital of Chongqing Medical University.

\section{Informed Consent: N/A.}

Peer-review: Externally peer-reviewed.

Author contributions: Concept - Y.W., D.L.; Design - D.J.; Supervision - W.L., D.J.; Resource - Y.W., D.L.; Materials - D.L., W.L., D.J.; Data Collection and/or Processing - Y.W., D.J.; Analysis and/or Interpretation - Y.W., D.L.; Literature Search - W.L.; Writing - Y.W.; Critical Reviews - W.L., D.J.

Conflict of Interest: No conflict of interest was declared by the authors.

Financial Disclosure: This study was funded by Inner Mongolia Science Programs (Project number: 2013MS11106).

\section{REFERENCES}

1. Dezateux C, Rosendahl K. Developmental dysplasia of the hip. Lancet 2007:369:1541-52. [CrossRef]

2. Guille JT, Pizzutillo PD, MacEwen GD. Development dysplasia of the hip from birth to six months. J Am Acad Orthop Surg 2000;8:232-42. [CrossRef]

3. Panagiotopoulou N, Bitar K, Hart WJ. The association between mode of delivery and developmental dysplasia of the hip in breech infants: a systematic review of 9 cohort studies. Acta Orthop Belg 2012;78:697-702.

4. Furnes O, Lie SA, Espehaug B, Vollset SE, Engesaeter LB, Havelin LI. Hip disease and the prognosis of total hip replacements. A review of 53,698 primary total hip replacements reported to the Norwegian arthroplasty register 1987-99. J Bone Joint Surg Br 2001;83:579-86. [CrossRef]

5. Chan A, McCaul KA, Cundy PJ, Haan EA, Byron-Scott R. Perinatal risk factors for developmental dysplasia of the hip. Arch Dis Child Neonatal Ed 1997;76:94-100. [CrossRef]

6. Sionek A, Czubak J, Kornacka K, Grabowski B. Evaluation of risk factors in developmental dysplasia of the hip in children from multiple pregnancies: results of hip ultrasonography using Graf's method. Ortop Traumatol Rehabil 2008;10:115-130.

7. Stein-Zamir C, Volovik, Rishpon S, Sabi R. Developmental dysplasia of the hip: risk markers, clinical screening and outcome. Pediatr Int 2008;50:341-5. [CrossRef]

8. Loening AM, James IE, Levenston ME, Badger AM, Frank EH, Kurz B, et al. Injurious mechanical compression of bovine articular cartilage induces chondrocyte apoptosis. Arch Biochem Biophys 2000;15:205-12. [CrossRef]

9. D'Lima DD, Hashimoto S, Chen PC, Colwell CW Jr, Lotz MK. Human chondrocyte apoptosis in response to mechanical injury. Osteoarthritis Cartilage 2001;9:712-9. [CrossRef]

10. Lotz M, Hashimoto S, Kühn K. Mechanisms of chondrocyte apoptosis. Osteoarthritis Cartilage 1999;7:389-91. [CrossRef]

11. Kouri JB, Aguilera JM, Reyes J, Lozoya KA, González S. Apoptotic chondrocytes from osteoarthrotic human articular cartilage and abnormal calcification of subchondral bone. J Rheumatol 2000;27:1005-19.

12. Hashimoto S, Ochs RL, Komiya S, Lotz M. Linkage of chondrocyte apoptosis and cartilage degradation in human osteoarthritis. Arthritis Rheum 1998;41:1632-8. [CrossRef]

13. Kim HA, Lee YJ, Seong SC, Choe KW, Song YW. Apoptotic chondrocyte death in human osteoarthritis. $J$ Rheumatol 2000;27:455-62.

14. Thomas CM, Fuller CJ, Whittles CE, Sharif Ml. Chondrocyte death by apoptosis is associated with cartilage matrix degradation. Osteoarthritis and Cartilage 2007;15:27-34. [CrossRef]

15. Shapiro F, Connolly S, Zurakowski D, Flynn E, Jaramillo D. Acetabular changes associated with avascular necrosis of the femoral head in a piglet model. Bone Joint Res 2014;3:130-8. [CrossRef]

16. Klisic PJ. Congenital dislocation of the hip-a misleading term: brief report. J Bone Joint Surg Br 1989;71:136. 
17. Aronsson DD, Goldberg MJ, Kling TF Jr, Roy DR. Developmental dysplasia of the hip. Pediatrics 1994;94:201-8.

18. Zemmyo M, Meharra EJ, Kühn K, Creighton-Achermann L, Lotz M. Accelerated, aging-dependent development of osteoarthritis in $\alpha 1$ integrin-deficient mice. Arthritis Rheum 2003;48:2873-80. [CrossRef]

19. Mocetti P, Silvestrini G, Ballanti P, Patacchioli FR, Di Grezia $\mathrm{R}$, Angelucci L, et al. Bcl-2 and Bax expression in cartilage and bone cells after high-dose corticosterone treatment in rats. Tissue Cell 2001;33:1-7. [CrossRef]

20. Islam N, Haqqi TM, Jepsen KJ, Kraay M, Welter JF, Goldberg $\mathrm{VM}$, et al. Hydrostatic pressure induces apoptosis in human chondrocytes from osteoarthritic cartilage through up-regulation of tumor necrosis factor-alpha, inducible nitric oxide synthase, p53, c-myc, and bax-alpha, and suppression of bcl-2. J Cell Biochem 2002;87:266-78. [CrossRef]

21. Fan TJ, Han LH, Cong RS, Liang J. Caspase family proteases and apoptosis. Acta Biochim Biophys Sin (Shanghai) 2005;37:71927. [CrossRef]

22. Kumar S. Caspase function in programmed cell death. Cell Death Differ 2007;14:32-43. [CrossRef]

23. Nicholson DW. Caspase structure, proteolytic substrates, and function during apoptotic cell death. Cell Death Differ 1999;6:1028-42. [CrossRef]

24. Kruidering M, Evan GI. Caspase-8 in apoptosis: the beginning of "the end"? IUBMB Life 2000;50:85-90. [CrossRef]

25. Porter AG, Janicke RU. Emerging roles of caspase-3 in apoptosis. Cell Death Differ 1999;6:99-104. [CrossRef] 